\title{
Trial registration 10 years on
}

\author{
The single most valuable tool we have to ensure unbiased reporting of research studies
}

\author{
Wim E J Weber European research editor, José G Merino US research editor, Elizabeth Loder \\ acting head of research
}

The BMJ

This month marks the tenth anniversary of the landmark decision by the International Committee of Medical Journal Editors to make journals require "registration of any clinical trials in a public trials registry at or before the time of first patient enrolment as a condition of consideration for publication."

It has been a long journey. The idea of prospective registration was first suggested in $1986,{ }^{2}$ but it took two decades for the idea to become reality. Since then both the United States and the European Union have passed legislation making registration a legal requirement for most types of clinical trials. Accordingly, the number of registered trials has gone up dramatically-ClinicalTrials.gov currently lists 189473 studies, the EU register has 25829 , and the Chinese Clinical Trial Registry has $5199 .^{3}$

As medical journal editors we are convinced that the requirement for prospective trial registration is the single most valuable tool we have to ensure unbiased reporting. It allows us to make sure that the published paper accurately reports the prespecified trial outcomes, samples sizes, and other planned analyses. It is the only way to identify outcome reporting bias and other deviations from the planned study to prevent such distortions from reaching publication. Before registration outcome reporting bias was widely accepted as being a major problem that deserved more attention. ${ }^{4}$ Research suggests that the requirement has improved the quality of reporting, ${ }^{5}$ although some disciplines seem to do better than others. ${ }^{67}$

Problems remain. Despite the registration requirements, The $B M J$ still receives a substantial number of papers reporting trials that were not prospectively registered. Since spring 2013 we have been keeping an informal tally of randomised trials submitted to The BMJ that were unregistered or registered late - as at 1 July, the list contained 69 entries. Most authors' explanations for non-compliance are not persuasive, and we reject those papers. The reasons cited for lack of compliance are varied, but at least two worrisome themes have emerged. Firstly, some senior authors do not take responsibility for ensuring prospective trial registration and identify junior study personnel as the reason registration did not occur-for example, stating that "our project coordinator was responsible for all registration related tasks."
Secondly, some academic authors believe that registration requirements should not or do not apply to trials that are not funded by industry, despite considerable evidence that publication bias affects all types of studies. ${ }^{8}$ Other explanations for non-registration that we have received from academic investigators include being too busy, lacking the resources needed to comply with regulations, or the belief that the rules should not apply to investigators working in less developed countries. One author asked us to overlook the failure to register a large foundation funded trial because the reason to require trial registration was "mainly to stop drug companies. [The principal investigator] is a developing country scientist doing this important study alongside a very busy job. Drug companies have whole departments devoted to compliance with regulations and processes like this."

Fortunately, the great majority of trials submitted to The BMJ are properly registered, and we do our best to ensure that the published report is in accordance with the registration. A recent study confirms that we are doing a reasonable job, although it is still far from perfect. ${ }^{9}$ The main problem is that it takes a substantial amount of editorial and reviewer time-large journals are staffed to do this, but smaller medical journals struggle. Recent research shows that many journals still do not adhere to the International Committee of Medical Journal Editors' trial registration policy. ${ }^{10}$ Others that do may be very generous in allowing exceptions. Even more worrisome, many editors do not feel that prospective registration and adherence to the registered plan is important. ${ }^{11}$ This is a worrying trend, because non-adherence is not confined to low impact journals. We need ways to enforce this requirement. Given that for many types of trials the policy is backed by legal requirements, this should be possible.

The trial registration process must be improved. Registries should implement better quality checks on data entry into the registry, as these are often inadequate. ${ }^{12}$ The time required to process registrations in some registries is too long, and this may lead to misclassification of some trials as retrospectively registered. Another way clinical registries can promote unbiased reporting of clinical trials is to accept (or even require) posting of the complete original protocol and statistical plan and any 
amendments, all with a time stamp. We have made this suggestion to the National Institutes of Health, when it asked for public comments on its draft policy to promote registration and results submission to ClinicalTrials.gov. ${ }^{13}$

Prospective registration of clinical trials is now an ethical and, in many cases, a legal requirement. Registries, public health agencies, funders, and journals must ensure compliance with these requirements to avoid biased and incomplete reporting of clinical research studies.

Competing interests: We have read and understood BMJ policy on declaration of interests and declare that EL has an academic interest in the topic of trial registration. WEJW and JGM have no relevant interests to declare.

Provenance and peer review: Commissioned, not externally peer reviewed

1 DeAngelis CD, Drazen JM, Frizelle FA, et al. Clinical trial registration: a statement from the International Committee of Medical Journal Editors. JAMA 2004;292:1363-4.

2 Simes RJ. Publication bias: the case for an international registry of clinical trials. J Clin Oncol 1986:4:1529-41.

3 ClinicalTrials.gov. Trends, charts, and maps. May 2015. https://clinicaltrials.gov/ct2/ resources/trends.
4 Mathieu S, Boutron I, Moher D, Altman DG, Ravaud P. Comparison of registered and published primary outcomes in randomized controlled trials. JAMA 2009;302:977-84.

5 To MJ, Jones J, Emara M, Jadad AR. Are reports of randomized controlled trials improving over time? A systematic review of 284 articles published in high-impact general and specialized medical journals. PLoS One 2013;8:e84779.

6 Killeen S, Sourallous P, Hunter IA, Hartley JE, Grady HL. Registration rates, adequacy of registration, and a comparison of registered and published primary outcomes in randomized controlled trials published in surgery journals. Ann Surg 2014;259:193-6.

7 Su CX, Han M, Ren J, et al. Empirical evidence for outcome reporting bias in randomized clinical trials of acupuncture: comparison of registered records and subsequent publications. Trials 2015;16:28.

8 Dwan K, Gamble C, Williamson PR, Kirkham JJ. Systematic review of the empirical evidence of study publication bias and outcome reporting bias-an updated review. PLOS One 2013;8:e66844.

9 van Lent M, IntHout J, Out HJ. Differences between information in registries and articles did not influence publication acceptance. J Clin Epidemiol 29 Nov 2014, doi:10.1016/j. jclinepi.2014.11.019.

10 Hooft L, Korevaar DA, Molenaar N, Bossuyt PM, Scholten RJ. Endorsement of ICMJE's clinical trial registration policy: a survey among journal editors. Neth J Med 2014:72:349-55.

11 Wager E, Williams P, Project Overcome Failure to Publish Negative Findings Consortium. "Hardly worth the effort"? Medical journals' policies and their editors' and publishers' views on trial registration and publication bias: quantitative and qualitative study. $B M J$ 2013;347:55248

12 Zarin DA, Tse T. Trust but verify: trial registration and determining fidelity to the protocol. Ann Intern Med 2013;159:65-7.

13 National Institutes of Health. NIH request for public comments on the draft $\mathrm{NIH}$ policy on dissemination of $\mathrm{NIH}$-funded clinical trial information. Nov 2014. http://grants.nih.gov/ grants/guide/notice-files/NOT-OD-15-019.html.

Cite this as: BMJ 2015;351:h3572

๑ BMJ Publishing Group Ltd 2015 